Draft version April 4, 2019

Typeset using $\mathrm{LAT}_{\mathrm{E}} \mathrm{X}$ twocolumn style in AASTeX62

\title{
RR LYRAE STARS IN THE FIELD OF SAGITTARIUS II
}

\author{
Seok-Joo Joo, ${ }^{1,2,3}$ Jaemann Kyeong, ${ }^{3}$ Soung-Chul Yang, ${ }^{3}$ Sang-Il Han, ${ }^{1,2,3}$ Eon-Chang Sung, ${ }^{3}$ Soo-Chang Rey, ${ }^{1}$ \\ Helmut Jerjen, ${ }^{4}$ Hak-Sub Kim, ${ }^{3}$ Dongwon Kim, ${ }^{5}$ Hyunjin Jeong, ${ }^{3}$ Chang H. Ree, ${ }^{3}$ Sang-Mok Cha, ${ }^{3,6}$ And \\ YONGSEOK LEE ${ }^{3,6}$ \\ ${ }^{1}$ Department of Astronomy and Space Science, Chungnam National University, 99 Daehak-ro, Daejeon 34134, Korea \\ ${ }^{2}$ Research Institute of Natural Sciences, Chungnam National University, 99 Daehak-ro, Daejeon 34134, Korea \\ ${ }^{3}$ Korea Astronomy and Space Science Institute, Daejeon 34055, Korea \\ ${ }^{4}$ Research School of Astronomy and Astrophysics, Australian National University, Canberra, ACT 2611, Australia \\ ${ }^{5}$ Department of Astronomy, University of California, Berkeley, CA 94720-3411, USA \\ ${ }^{6}$ School of Space Research, Kyung Hee University, Yongin, Kyeonggi 17104, Korea
}

\begin{abstract}
We present the detection of RR Lyrae variable stars in the field of the Sagittarius II (Sgr II) ultrafaint dwarf (UFD) galaxy. Using $B, V$ time-series photometry obtained with the Korea Microlensing Telescope Network (KMTNet) $1.6 \mathrm{~m}$ telescope at CTIO and $G$-band data from Gaia Data Release 2 (DR2), we identified and characterized two $a b$-type and four $c$-type RR Lyrae variables. Five out of the six stars are clustered within three half-light radii $(\sim 4 ! 8)$ of the galaxy indicating their association with Sgr II, while the RRab star V4 is located $\sim 22^{\prime}$ from the galaxy center. By excluding V4, the high $c$-type fraction (0.8) and the period of the only RRab star V3 ( $P_{V 3} \simeq 0.666$ days) suggest an Oosterhoff II (Oo II) classification for Sgr II. Located close to the locus of Oo II clusters in the periodamplitude diagram, V3 is similar to RRab stars in other UFDs having Oosterhoff-intermediate and Oo II properties. Sgr II is, however, more compact than usual UFDs, placed in between star clusters and dwarf galaxies in the size-luminosity plane, and therefore spectroscopic studies are eventually required to ascertain the true nature of this stellar system. We derive the metallicity $\left([\mathrm{Fe} / \mathrm{H}]_{\mathrm{RRab}} \simeq-2.1 \pm 0.3\right)$ and heliocentric distance ( $\sim 64 \pm 3 \mathrm{kpc})$ of Sgr II from the RR Lyrae stars, and estimate its age $(\sim 12 \mathrm{Gyr})$ based on our stellar population models. The Oosterhoff properties of UFDs can be explained with the evolution effect of RR Lyrae stars in the instability strip.
\end{abstract}

Keywords: galaxies: dwarf — galaxies: individual (Sagittarius II) — Local Group — stars: variables: RR Lyrae

\section{INTRODUCTION}

Since 2005, about 40 dwarf satellite galaxies have been discovered around the Milky Way (MW) from large optical surveys such as SDSS (York et al. 2000), DES (DES Collaboration 2016), and Pan-STARRS1 (Chambers et al. 2016), greatly exceeding the number of twelve classically known dwarf satellites. Most of the new stellar systems are ultra-faint dwarfs (UFDs), the smallest and least luminous galaxies known. Expected to be completely dark matter dominated (e.g. Simon \& Geha 2007), detailed investigations of these UFDs are of particular importance to better understand the formation and evolution of galaxies in the

sjjoo250@gmail.com current $\Lambda$ CDM hierarchical merging paradigm (e.g., Kauffmann et al. 1993).

RR Lyrae stars provide an excellent opportunity to study resolved stellar populations. They are well-known standard candles and tracers of old, metal-poor populations. Metallicity and line-of-sight reddening can also be derived from their variability characteristics. By comparing them with those in other systems including the MW halo, we can place constraints on the formation and evolution of the MW and dwarf galaxies (e.g., Clementini 2010). Thus far, about 15 UFDs with total luminosities $M_{V}>-7$ have been studied for RR Lyrae stars, and it was reported that all of them have at least one RR Lyrae star (e.g., Vivas et al. 2016), with the only exception of Carina III (Torrealba et al. 2018).

In this paper, we investigate RR Lyrae stars in the field of the Sagittarius II (Sgr II) dwarf galaxy, as part 
of our ongoing time-series study of dwarf satellites in the southern hemisphere using the Korea Microlensing Telescope Network (KMTNet) $1.6 \mathrm{~m}$ telescope at Cerro Tololo Inter-American Observatory (CTIO). Recently discovered by Laevens et al. (2015) from the PanSTARRS1 survey, Sgr II has a total luminosity of $M_{V} \simeq$ -5.2 and a heliocentric distance of $\sim 67 \mathrm{kpc}$ (see also Mutlu-Pakdil et al. 2018). Compared to typical UFDs with similar luminosities, it is relatively small and compact (half-light radius, $r_{h} \approx 32 \mathrm{pc}$ or 1.6 ), but is larger than usual globular clusters (GCs). In the sizeluminosity plane, Sgr II can be classified as either the most compact UFD or one of the most extended GCs. For that reason, Laevens et al. (2015) also assigned an alternative name (Laevens 5) for this stellar system. We identify and analyze RR Lyrae variables by fitting light curve templates to our time-series photometry. The data from Gaia Data Release 2 (DR2)(Gaia Collaboration et al. 2016, 2018) is also used to complement our data. We compare them with those stars of other UFDs in the period-amplitude diagram and discuss the implications of our results.

\section{OBSERVATIONS, DATA REDUCTION, AND COLOR-MAGNITUDE DIAGRAMS}

Time-series observations of the field of Sgr II were conducted in the $B$ - and $V$-bands using the KMTNet-CTIO $1.6 \mathrm{~m}$ telescope on 17 nights from October to November in 2016 and from September to October in 2017. In total, 117 and 121 frames were obtained for $B$ - and $V$-bands, respectively, with an exposure time of $120 \mathrm{~s}$ each. Point-spread function (PSF) photometry was performed using DAOPHOT II/ALLSTAR (Stetson 1987) and ALLFRAME (Stetson 1994) on the time-series data preprocessed by the KMTNet pipeline (Kim et al. 2016). We used the AAVSO Photometric All-Sky Survey (APASS) database (http://www.aavso.org/apass) for standardization and SCAMP (Bertin 2006) for astrometry by adopting the third US Naval Observatory (USNO) CCD Astrograph Catalog, UCAC3 (Zacharias et al. 2010). We also used the time-series data from Gaia DR2 (Gaia Collaboration et al. 2018) for stars identified as variables in the field of Sgr II.

In the left and middle panels of Figure 1, we present color-magnitude diagrams (CMDs) of the Sgr II field, for the region inside $r_{h}(\sim 1 ! 6$; Mutlu-Pakdil et al. 2018) and $3 r_{h}(\sim 4 ! 8)$, respectively. To obtain the CMDs, we combined our time-series images with SWarp (Bertin et al. 2002) and carried out PSF photometry with DAOPHOT II/ALLSTAR (Stetson 1987). In both panels, blue horizontal branch $(\mathrm{HB})$ and red giant branch (RGB) stars of Sgr II are prominent over the background of field stars. The CMD features of Sgr II are comparable to those presented in Laevens et al. (2015, see their Figure 1) and Mutlu-Pakdil et al. (2018, see their Figure 1), while the CMD in the latter is much deeper than ours. RR Lyrae stars identified in this study are also plotted as crosses in the panels (see next section). In the right panel, we compare our population models with the foreground-subtracted Hess diagram for $2 r_{h}(r<3 ! 2)$ region, which will be discussed in Section 4.

\section{RR LYRAE STARS IN THE FIELD OF SGR II}

RR Lyrae variables in the field of Sgr II were detected and characterized using the template light curve fitting technique outlined by Yang \& Sarajedini (2012) and Yang et al. (2014). We selected variable candidates by examining the variability of stars at the level of the HB, and analyzed them by fitting template light curves. Readers are referred to Joo et al. (2018, hereafter Paper I) for details of the procedure we employed. Finally, six RR Lyrae (two RRab and four RRc) stars were discovered in the Sgr II field. We found that five of the six variables are also identified as RR Lyrae stars in Gaia DR2 (Gaia Collaboration et al. 2018). For these five stars, V2 to V6, we included the Gaia time-series data in our light curve analysis by transforming the Gaia $G$-band to $V$-band magnitudes based on the relations in Table A2 of Evans et al. (2018, see also Clementini et al. 2019). The light curves obtained with our best-fit templates are presented in Figure 2, where the green points are the Gaia $G$-band data converted to $V$-band. In Table 1, we summarize positions and pulsation properties of RR Lyrae stars including variable type, period, intensity-weighted mean magnitude, the number of observations, and amplitude. We also list the information (ID, type, period, and the number of data points) from Gaia DR2 for the five variables matched with our data. Note that the type and period values from Gaia DR2 are almost identical to those estimated in our analysis.

The left panel of Figure 3 is the CMD showing a closeup view of the HB region for stars within 4'.8 from the galaxy center. The six RR Lyrae stars detected in this study are well located in the empirical instability strip, which is estimated from the data of nine Galactic and Large Magellanic Cloud clusters in Walker (1998, Table 7 in that paper). We see that V1 is classified as $c$-type from the light-curve shape and short period, while it is the reddest RR Lyrae in the CMD. This star appears to be slightly affected by bleeding from a saturated star, roughly up to $\sim 0.2 \mathrm{mag}$ more in $V$-band than $B$-band. If this effect is taken into account, the $B-V$ color of V1 would be similar to those of the other $c$-type variables. 
The middle panel of Figure 3 presents the spatial distribution of the RR Lyrae and blue HB stars, while the right panel displays that of all point sources detected in the field for comparison. The RR Lyrae stars are clearly clustered around the galaxy center estimated by Mutlu-Pakdil et al. (2018). Five out of the six RR Lyrae stars are within $3 r_{h}$ (i.e., $r<4$ ! 8 ), while the RRab star $\mathrm{V} 4$ is located $\sim 22^{\prime}$ away from the center. The blue HB stars, that we define as stars in the color and magnitude ranges of $0.0<B-V<0.27$ and $19.5<V<20.3$, are also centrally concentrated. We do not find, however, such a clustering of stars belonging to the red HB region, i.e., $0.50<B-V<0.65$ at the same magnitude range. This suggests that there are only a few red HB stars in Sgr II, if any, indicating the blue HB morphology of Sgr II in conjunction with the CMDs in Figure 1.

The positions in the CMD and the spatial distribution of the RR Lyrae and blue HB stars strongly indicate that most of these stars near the center are associated with Sgr II. For the stars outside $\sim 6 r_{h}$ (i.e., $r \gtrsim 9$ '.6) including V4, it is natural to consider them as field stars, given the heliocentric distance to the galaxy $(\sim 64 \mathrm{kpc}$, see below). By excluding the $a b$-type star V4, the fraction of $c$-type RR Lyrae variables is calculated to be, $N(c) / N(a b+c)=\frac{4}{5}=0.8$. This high fraction of RRc stars is also consistent with the blue HB morphology of the system. The period of the only $a b$-type star in Sgr II, V3, is $P_{a b} \simeq 0.666$ days, and the mean period of the four $c$-type stars is $\left\langle P_{c}\right\rangle=0.337 \pm 0.023$ days, where the uncertainty is the standard error of the mean. Note that the long period of the RRab star V3 and the high $c$-type fraction suggest an Oosterhoff II (Oo II) classification $^{1}$ for Sgr II (see, e.g., Catelan 2009). The mean apparent $V$ magnitude of the RR Lyrae stars is $\left\langle V_{\mathrm{RR}}\right\rangle=19.77 \pm 0.04 \mathrm{mag}$, where we also exclude V1, which is affected by bleeding, as well as V4, and the uncertainty is the standard error of the mean.

Figure 4 shows the period-amplitude diagram of the RR Lyrae stars in the Sgr II field, and compares them with those in the MW halo and $13 \mathrm{UFDs}^{2}$ with $M_{V}>$ -7 , as an update of Figure 10 in Vivas et al. (2016) and Figure 6 in Paper I. The solid and dotted lines rep-

\footnotetext{
1 Note however that the Oosterhoff classification is basically based on the mean period of RRab stars and therefore usually applied to the systems hosting at least five RRab stars (see, e.g., Catelan 2009). Braga et al. (2016) have further shown that, using Galactic GCs with more than 35 RR Lyrae stars and nearby dwarf galaxies, the difference in the mean RR Lyrae periods between the Oosterhoff groups (i.e., Oosterhoff dichotomy) can be explained by changes in metallicity.

${ }^{2}$ Segue 1 is not included here because of the absence of accurate period and amplitude information, while Simon et al. (2011) has reported one or two RR Lyrae stars (see also Vivas et al. 2016).
}

resent the mean distribution of RR Lyrae stars in the MW GCs that belong to the Oosterhoff I (Oo I) and Oo II groups, respectively, adopted from Zorotovic et al. (2010) and Cacciari et al. (2005). It is clear from Figure 4 that RRab stars in the UFDs studied so far have mostly Oosterhoff-intermediate (Oo-int) or Oo II properties (see also Clementini 2010; Vivas et al. 2016), in contrast to those in the MW halo where the majority $(\sim 73 \%)$ has Oo I properties (Zinn et al. 2014). Placed close to the locus of Oo II clusters, V3 is in the middle of the distribution of stars in the UFDs in terms of both period and amplitude. ${ }^{3} \mathrm{~V} 4$ is, however, almost on the Oo I line, quite different from most RRab stars in the UFDs. This supports the previous assumption based on its spatial location that V4 is not a member of Sgr II but belongs to the MW halo. We can then conclude that Sgr II is also similar to the other UFDs in the distribution of RRab stars on the period-amplitude diagram and is classified as Oo II. ${ }^{1}$

The blue HB morphology of Sgr II and the similarity in RR Lyrae properties between Sgr II and other UFDs suggest that Sgr II can be considered as a UFD, because outer halo GCs $(\gtrsim 40 \mathrm{kpc})$ tend to have red HB morphologies (e.g, Lee et al. 1994; Laevens et al. 2015). However, this does not mean that Sgr II cannot be a GC. There are GCs with Oo II characteristics similar to Sgr II in the inner halo (Clement et al. 2001), and Mutlu-Pakdil et al. (2018) has further shown that its structural parameters are more consistent with extended GCs. Spectroscopic studies are eventually required to ascertain whether it is a galaxy or a star cluster, by investigating velocity and metallicity dispersions of the system and/or a possible presence of $\mathrm{Na}-\mathrm{O}$ or $\mathrm{Mg}-\mathrm{Al}$ anti-correlations shown only in GCs.

Using the properties of the RRab star V3, we derive the interstellar reddening in the direction and metallicity of Sgr II. From the empirical period-amplitudemetallicity relation by Alcock et al. (2000, see equations (1) and (2) in that paper), $[\mathrm{Fe} / \mathrm{H}]=-2.1 \pm 0.3$ is obtained, where the uncertainty reflects the intrinsic accuracy of the relation. This is in good agreement with the estimate $([\mathrm{Fe} / \mathrm{H}] \approx-2.2)$ from isochrone fitting by Laevens et al. (2015). From Sturch's method (Sturch 1966) revised by Walker (1990, see equation (2) in that paper) and the (period-)amplitude-color-metallicity relation by Piersimoni et al. (2002, see equations (4) and (5) in Paper I), we infer $E(B-V) \approx 0.18$ and 0.15 , respectively. These $E(B-V)$ values are, however, some-

\footnotetext{
$3 \quad$ V3 is also consistent with the findings that faint dwarf spheroidal galaxies and UFDs show a lack of high-amplitude shortperiod RRab stars (e.g., Fiorentino et al. 2017).
} 
what larger than that of Laevens et al. (2015), 0.097, measured from the dust maps of Schlegel et al. (1998) and Schlafly \& Finkbeiner (2011). Because the reddening value from RRab stars strongly depends on the observed color (or color distribution) of them, we have less confidence in our reddening estimation based on a single RRab star. Instead, by adopting $E(B-V)=0.097$ to the instability strip, we obtain a reasonable match with the observed mean colors of the RR Lyrae stars in the CMD (see Figure 3a).

We also estimate the distance to Sgr II using optical Period-Wesenheit (PW) relations provided by Marconi et al. (2015), which have the key advantage that they are reddening free and only marginally dependent on metallicity (see also Bono et al. 2019, for a recent application of the $\mathrm{PW}$ relations). In practice, we adopt metal-independent $(V, B-V)$ PW relations (Marconi et al. 2015, see their equation (10) and Table 9) for the four RR Lyrae stars (excluding $\mathrm{V} 1$ and V4), and obtain the mean distance modulus, $(m-M)_{0}=19.03 \pm 0.10 \mathrm{mag}$, and the mean heliocentric distance, $\mathrm{d}_{\odot}=64 \pm 3 \mathrm{kpc}$, for Sgr II. The uncertainties are standard deviations. Our distance estimate agrees with those presented by Laevens et al. (2015, $67 \pm 5 \mathrm{kpc})$ and Mutlu-Pakdil et al. $(2018,70.2 \pm 5.0 \mathrm{kpc})$ to within the uncertainty. In the case of the probable field RRab star V4, a distance modulus of $\sim 18.58 \mathrm{mag}$ and a distance of $\sim 52 \mathrm{kpc}$ are obtained with the same relations, which implies that V4 is $\sim 12 \mathrm{kpc}$ closer than the center of Sgr II.

\section{DISCUSSION}

To estimate the age of the stellar population in Sgr II, we have constructed population models using Yonsei-Yale $\left(\mathrm{Y}^{2}\right)$ isochrones and $\mathrm{HB}$ evolutionary tracks (Yi et al. 2008; Han et al. 2009), based on the techniques developed by Lee et al. (1990, 1994) and Joo \& Lee (2013). With the fixed metallicity of $[\mathrm{Fe} / \mathrm{H}]=-2.1$ and alpha-element enhancement of $[\alpha / \mathrm{Fe}]=0.3$, the age value was adjusted until the model best matches the observed CMD. Our best-fit model with an age of 12 Gyr is presented in the right panel of Figure 1. Given the large observational errors near the main sequence turn-off (MSTO), the uncertainty in this age determination can be relatively large, ${ }^{4}$ but an old age is clearly required to reproduce the blue

\footnotetext{
4 The quadratic sum of the errors in the $V$-band magnitude and extinction (i.e., reddening) is $\sim 0.12 \mathrm{mag}$ at the MSTO, which means that the age uncertainty caused by the photometric errors could be more than $1 \mathrm{Gyr}$.
}

HB morphology of Sgr II, which is comparable to those of other UFDs (e.g., Brown et al. 2014).

Almost all UFDs (with $M_{V}>-7$ ) studied for RR Lyrae stars so far have Oo-int or Oo II properties, as shown in Figure 4. If these UFDs host a purely old and metal-poor population resulting in a substantially blue $\mathrm{HB}$ morphology, this can be explained with the effect of evolution of RR Lyrae stars in the instability strip. Because in that situation most RRab variables are highly evolved stars from the blue side of the instability strip, they are expected to have longer periods than those at the zero-age HB phase (e.g., Lee et al. 1990). The relatively long period of V3 in Sgr II can also be explained by this evolution effect.

Another interesting property of Sgr II is that, it is at the expected location of the trailing arm of the Sgr stream yet to be detected behind the Sgr dwarf galaxy and therefore it might be originally a satellite of the Sgr galaxy but brought into the MW halo, as suggested by Laevens et al. (2015). While we could not find evidence for that hypothesis from RR Lyrae stars, if confirmed, it would mean that the properties of UFD satellites belonging to the MW halo and the Sgr galaxy are similar in terms of their stellar populations (i.e., age and metallicity). Combined with the expected association with the Magellanic Clouds of some UFDs in the southern hemisphere (e.g., Jethwa et al. 2016; Jerjen et al. 2018), this would further imply universal properties of UFDs in the universe as relics of the first galaxies and/or surviving counterparts of the basic building blocks that merged and disrupted to form larger galaxies.

We thank the anonymous referee for a number of helpful comments and suggestions. This research has made use of the KMTNet system operated by the Korea Astronomy and Space Science Institute (KASI) and the data were obtained at one of three host sites, CTIO in Chile. This work has made use of data from the European Space Agency (ESA) mission Gaia (https://www.cosmos.esa.int/gaia), processed by the Gaia Data Processing and Analysis Consortium (DPAC, https://www.cosmos.esa.int/web/gaia/dpac/consortium). Funding for the DPAC has been provided by national institutions, in particular the institutions participating in the Gaia Multilateral Agreement. S.C.R was partially supported by the Basic Science Research Program through the NRF of Korea funded by the Ministry of Education (2018R1A2B2006445). Support for this work was also provided by the NRF to the Center for Galaxy Evolution Research (2017R1A5A1070354). H.Jerjen acknowledges the support of the Australian Research Council through Discovery Project DP150100862. 
H.Jeong acknowledges support from the Basic Science Research Program through the National Research Foundation (NRF) of Korea, funded by the Ministry of Education (NRF-2013R1A6A3A04064993). This research was made possible through the use of the APASS, funded by the Robert Martin Ayers Sciences Fund.

\section{REFERENCES}

Alcock, C., Allsman, R. A., Alves, D. R., et al. 2000, AJ, 119, 2194

Bertin, E. 2006, in ASP Conf. Ser. 351, Astronomical Data Analysis Software and Systems XV, ed. C. Gabriel et al. (San Francisco, CA: ASP), 112

Bertin, E., Mellier, Y., Radovich, M., et al. 2002, in ASP Conf. Ser. 281, Astronomical Data Analysis Software and Systems XI, ed. D. A. Bohlender, D. Durand, \& T. H. Handley (San Francisco, CA: ASP), 228

Boettcher, E., Willman, B., Fadely, R., et al. 2013, AJ, 146, 94

Bono, G., Iannicola, G., Braga, V. F., et al. 2019, ApJ, 870, 115

Braga, V. F., Stetson, P. B., Bono, G., et al. 2016, AJ, 152, 170

Brown, T. M., Tumlinson, J., Geha, M., et al. 2014, ApJ, 796, 91

Cacciari, C., Corwin, T. M., \& Carney, B. W. 2005, AJ, 129,267

Catelan, M. 2009, Ap\&SS, 320, 261

Chambers, K. C., Magnier, E. A., Metcalfe, N., et al. 2016, arXiv:1612.05560

Clement, C. M., Muzzin, A., Dufton, Q., et al. 2001, AJ, 122,2587

Clementini, G. 2010, in Variable Stars, the Galactic Halo and Galaxy Formation, ed. N. Samus, C. Sterken, \& L. Szabados (Moscow: Sternberg Astronomical Institute of Moscow Univ.), 107

Clementini, G., Ripepi, V., Molinaro, R., et al. 2019, A\&A, 622, A60

Dall'Ora, M., Kinemuchi, K., Ripepi, V., et al. 2012, ApJ, 752,42

DES Collaboration 2016, MNRAS, 460, 1270

Dorfi, E. A., \& Feuchtinger, M. U. 1999, A\&A, 348, 815

Evans, D. W., Riello, M., De Angeli, F., et al. 2018, A\&A, 616, A4

Fiorentino, G., Monelli, M., Stetson, P. B., et al. 2017, A\&A, 599, A125

Gaia Collaboration, Brown, A. G. A., Vallenari, A., et al. 2018, A\&A, 616, A1

Gaia Collaboration, Prusti, T., de Bruijne, J. H. J., et al. 2016, A\&A, 595, A1

Garling, C., Willman, B., Sand, D. J., et al. 2018, ApJ, 852, 44
Garofalo, A., Cusano, F., Clementini, G., et al. 2013, ApJ, 767,62

Greco, C., Dall'Ora, M., Clementini, G., et al. 2008, ApJL, $675, \mathrm{~L} 73$

Han, S.-I., Kim, Y.-C., Lee, Y.-W., et al. 2009, in ESO Astrophysics Symposia, Globular Clusters - Guides to Galaxies, ed. T. Richtler \& S. Larsen (Berlin:Springer), 33

Holl, B., Audard, M., Nienartowicz, K., et al. 2018, A\&A, 618, A30

Jerjen, H., Conn, B., Kim, D., \& Schirmer, M. 2018, arXiv:1809.02259

Jester, S., Schneider, D. P., Richards, G. T., et al. 2005, AJ, 130, 873

Jethwa, P., Erkal, D., \& Belokurov, V. 2016, MNRAS, 461, 2212

Joo, S.-J., Kyeong, J., Yang, S.-C., et al. 2018, ApJ, 861, 23 (Paper I)

Joo, S.-J., \& Lee, Y.-W. 2013, ApJ, 762, 36

Kauffmann, G., White, S. D. M., \& Guiderdoni, B. 1993, MNRAS, 264, 201

Kim, S.-L., Lee, C.-U., Park, B.-G., et al. 2016, JKAS, 49, 37

Laevens, B. P. M., Martin, N. F., Bernard, E. J., et al. 2015, ApJ, 813, 44

Lee, Y.-W., Demarque, P., \& Zinn, R. 1990, ApJ, 350, 155

Lee, Y.-W., Demarque, P., \& Zinn, R. 1994, ApJ, 423, 248

Marconi, M., Coppola, G., Bono, G., et al. 2015, ApJ, 808, 50

Medina, G. E., Muñoz, R. R., Vivas, A. K., et al. 2017, ApJL, 845, L10

Moretti, M. I., Dall'Ora, M., Ripepi, V., et al. 2009, ApJL, 699, L125

Musella, I., Ripepi, V., Clementini, G., et al. 2009, ApJL, 695, L83

Musella, I., Ripepi, V., Marconi, M., et al. 2012, ApJ, 756, 121

Mutlu-Pakdil, B., Sand, D. J., Carlin, J. L., et al. 2018, ApJ, 863, 25

Piersimoni, A. M., Bono, G., \& Ripepi, V. 2002, AJ, 124, 1528

Schlafly, E. F., \& Finkbeiner, D. P. 2011, ApJ, 737, 103

Schlegel, D. J., Finkbeiner, D. P., \& Davis, M. 1998, ApJ, 500,525 
Sesar, B., Banholzer, S. R., Cohen, J. G., et al. 2014, ApJ, 793,135

Siegel, M. H. 2006, ApJL, 649, L83

Simon, J. D., \& Geha, M. 2007, ApJ, 670, 313

Simon, J. D., Geha, M., Minor, Q. E., et al. 2011, ApJ, 733, 46

Stetson, P. B. 1987, PASP, 99, 191

Stetson, P. B. 1994, PASP, 106, 250

Sturch, C. 1966, ApJ, 143, 774

Torrealba, G., Belokurov, V., Koposov, S. E., et al. 2018, MNRAS, 475, 5085

Vivas, A. K., Olsen, K., Blum, R., et al. 2016, AJ, 151, 118

Walker, A. R. 1990, AJ, 100, 1532

Walker, A. R. 1998, AJ, 116, 220
Yang, S.-C., \& Sarajedini, A. 2012, MNRAS, 419, 1362

Yang, S.-C., Wagner-Kaiser, R., Sarajedini, A., Kim, S. C., \& Kyeong, J. 2014, ApJ, 784, 76

Yi, S. K., Kim, Y.-C., Demarque, P., et al. 2008, IAU Symp. 252, The Art of Modeling Stars in the 21st Century, ed. L. Deng \& K.-L. Chan (Cambridge: Cambridge Univ. Press), 413

York, D. G., Adelman, J., Anderson, J. E., Jr., et al. 2000, AJ, 120, 1579

Zacharias, N., Finch, C., Girard, T., et al. 2010, AJ, 139, 2184

Zinn, R., Horowitz, B., Vivas, A. K., et al. 2014, ApJ, 781, 22

Zorotovic, M., Catelan, M., Smith, H. A., et al. 2010, AJ, 139,357 
Table 1. Pulsation Properties of RR Lyrae Stars in Sgr II

\begin{tabular}{|c|c|c|c|c|c|c|c|c|c|c|c|c|c|c|}
\hline \multirow[t]{3}{*}{ ID } & \multirow{3}{*}{$\begin{array}{l}\text { R.A. } \\
\text { (2000) }\end{array}$} & \multirow{3}{*}{$\begin{array}{l}\text { Dec. } \\
(2000)\end{array}$} & \multirow[t]{3}{*}{ Type } & \multirow{3}{*}{$\begin{array}{l}\text { Period } \\
\text { (days) }\end{array}$} & \multirow{3}{*}{$\begin{array}{c}\langle B\rangle^{\mathrm{i}} \\
(\mathrm{mag})\end{array}$} & \multirow{3}{*}{$\begin{array}{c}\langle V\rangle^{\mathrm{i}} \\
(\mathrm{mag})\end{array}$} & \multirow[t]{3}{*}{$N_{B}{ }^{\mathrm{ii}}$} & \multirow[t]{3}{*}{$N_{V}{ }^{\mathrm{ii}}$} & \multirow{3}{*}{$\begin{array}{l}A_{B}{ }^{\mathrm{iii}} \\
\text { (mag) }\end{array}$} & \multirow{3}{*}{$\begin{array}{l}A_{V}{ }^{\mathrm{iii}} \\
(\mathrm{mag})\end{array}$} & \multicolumn{4}{|c|}{ Gaia DR2 } \\
\hline & & & & & & & & & & & ID & Type & Period & $N_{G}^{\text {iv }}$ \\
\hline & & & & & & & & & & & & & (days) & \\
\hline V1 & $19: 52: 45.48$ & $-22: 02: 27.88$ & $\mathrm{c}$ & 0.3148 & 20.13 & 19.64 & 117 & 121 & 0.78 & 0.53 & $6864423852970123520^{v}$ & - & - & - \\
\hline $\mathrm{V} 2$ & $19: 52: 56.75$ & $-22: 04: 08.36$ & c & 0.4065 & 20.07 & 19.68 & 116 & 121 & 0.56 & 0.39 & 6864422757758521984 & $\mathrm{c}$ & 0.4065 & 32 \\
\hline V3 & $19: 52: 44.40$ & $-22: 03: 04.71$ & $a b$ & 0.6656 & 20.22 & 19.76 & 117 & 121 & 1.08 & 0.80 & 6864422993976659968 & $a b$ & 0.6657 & 34 \\
\hline $\mathrm{V} 4^{\mathrm{vi}}$ & $19: 52: 13.13$ & $-21: 42: 58.93$ & $a b$ & 0.5408 & 19.95 & 19.52 & 117 & 121 & 1.47 & 1.04 & 6865195302117134336 & $a b$ & 0.5407 & 37 \\
\hline V5 & $19: 52: 38.05$ & $-22: 03: 30.45$ & $\mathrm{c}$ & 0.3078 & 20.13 & 19.85 & 117 & 121 & 0.65 & 0.50 & 6864048408410304896 & $\mathrm{c}$ & 0.3079 & 36 \\
\hline V6 & $19: 52: 35.88$ & $-22: 01: 59.81$ & $\mathrm{c}$ & 0.3186 & 20.12 & 19.81 & 117 & 121 & 0.79 & 0.57 & 6864423994704275200 & $\mathrm{c}$ & 0.3186 & 35 \\
\hline
\end{tabular}

i Intensity-weighted mean magnitude. V1 is slightly contaminated by bleeding from a saturated star.

${ }^{\text {ii }}$ Number of observations used in our light curve analysis.

iii Pulsation amplitude.

${ }^{\text {iv }}$ Number of data points for G-band in Gaia DR2

${ }^{\mathrm{V}}$ Not identified as a variable in Gaia DR2.

${ }^{\mathrm{vi}}$ Field?

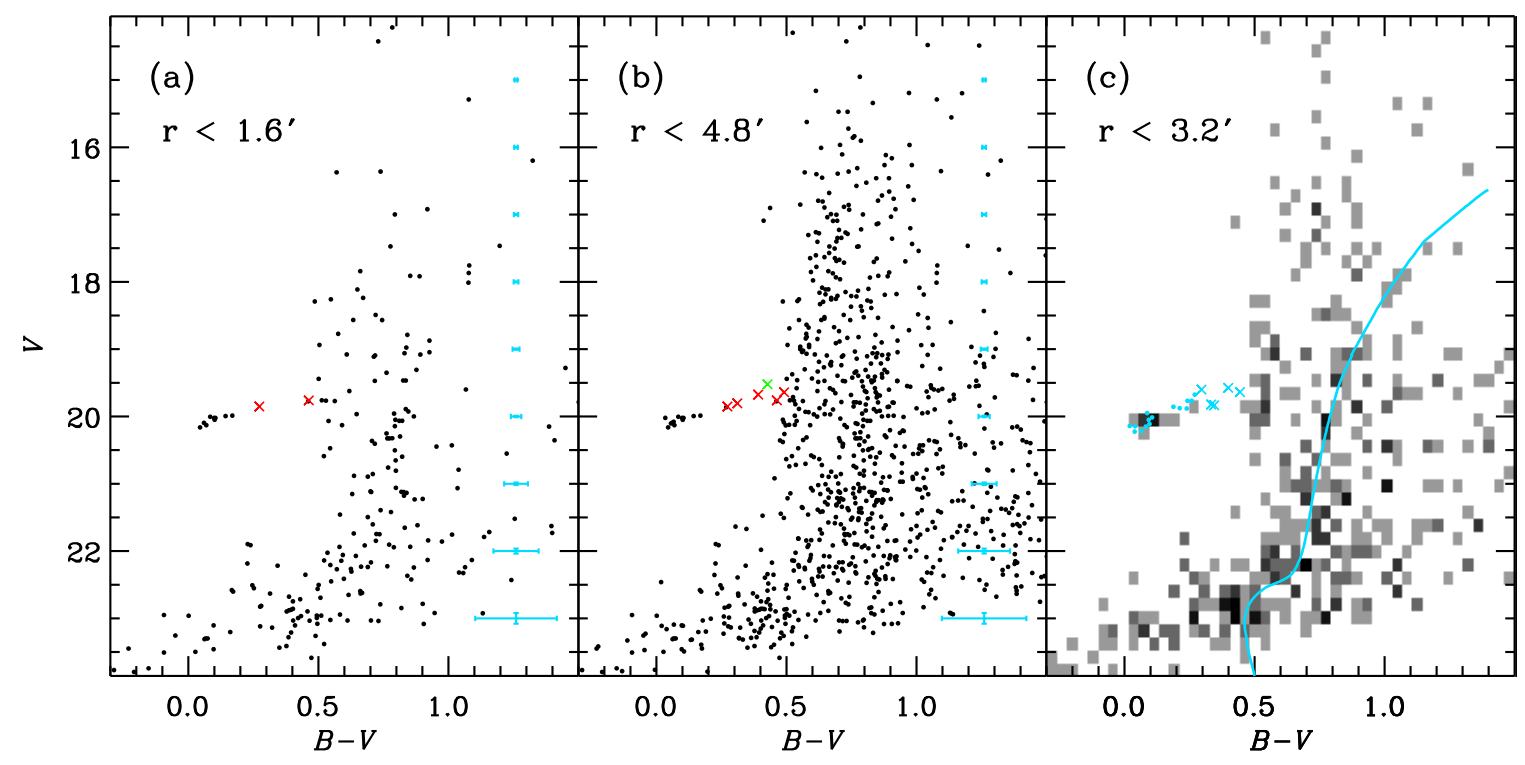

Figure 1. (a) and (b) CMD of stars within 1.'6 $\left(r_{h}\right)$ and $4{ }^{\prime} .8\left(3 r_{h}\right)$ regions in the field of Sgr II, respectively. Detected RR Lyrae variables are highlighted as crosses, while the green cross denotes V4, which is $\sim 22^{\prime}$ from the center. The cyan error bars represent the measurement errors. (c) Our population models (cyan line and points) superimposed on the foreground-subtracted Hess diagram for 3'.2 $\left(2 r_{h}\right)$ region. The foreground field was assumed to be an annulus of 9.'6 $\left(6 r_{h}\right)<r<13$.'9 (covering 10 times the area of $\left.2 r_{h}\right)$ and normalized. Model RR Lyrae stars are presented as cyan crosses. Adopted parameters are $t=12 \mathrm{Gyr}$, $[\mathrm{Fe} / \mathrm{H}]=-2.1,[\alpha / \mathrm{Fe}]=0.3, E(B-V)=0.097$, and $(m-M)_{0}=19.03 \mathrm{mag}$, respectively. 

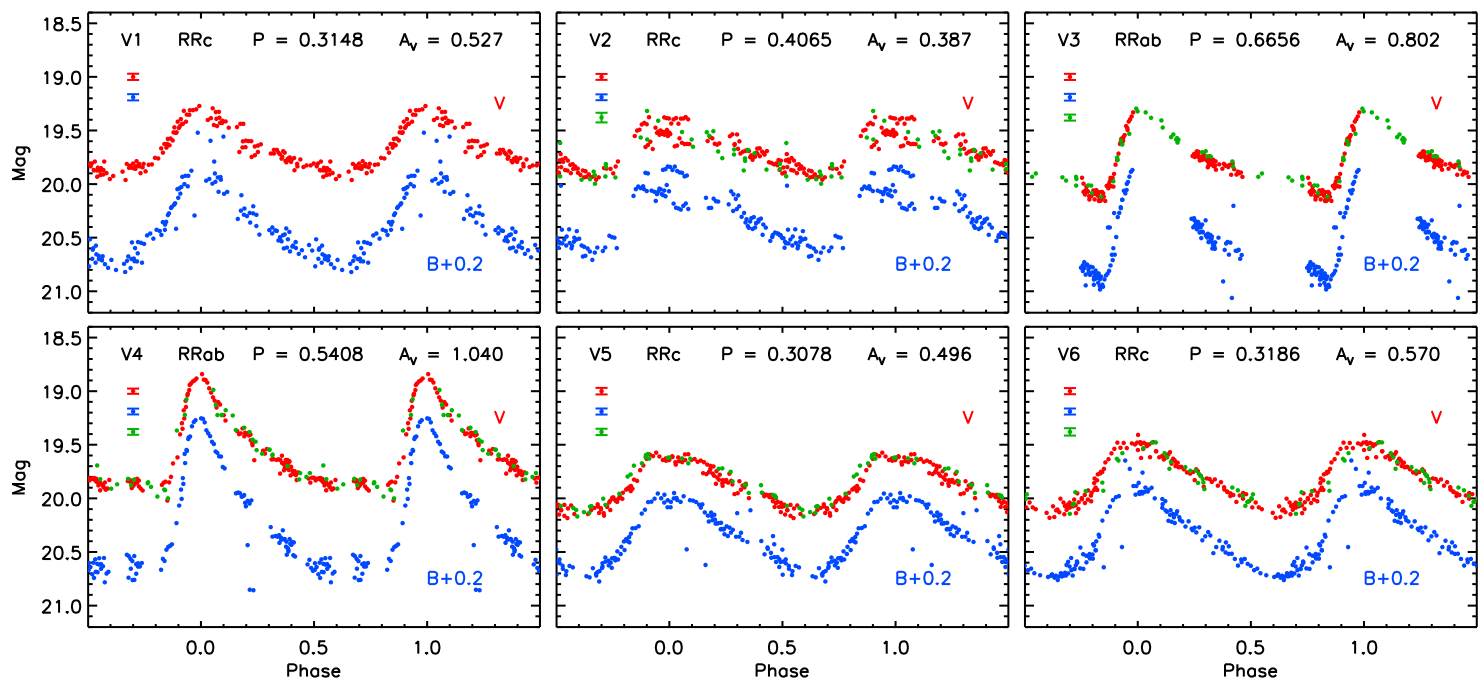

Figure 2. Light curves of the six RR Lyrae stars for $B$ - and $V$-bands (blue and red points), respectively. The $B$-band light curves are shifted by $+0.2 \mathrm{mag}$. Green points are the Gaia $G$-band data transformed to $V$-band magnitudes. The variable information including ID, type, period (days), and $V$-band amplitude (mag) is also presented in each panel. The error bars in the upper left corner are the mean photometric errors for each band. The $G$-band uncertainties are estimated using the relation in Holl et al. (2018, see their footnote 6 ).
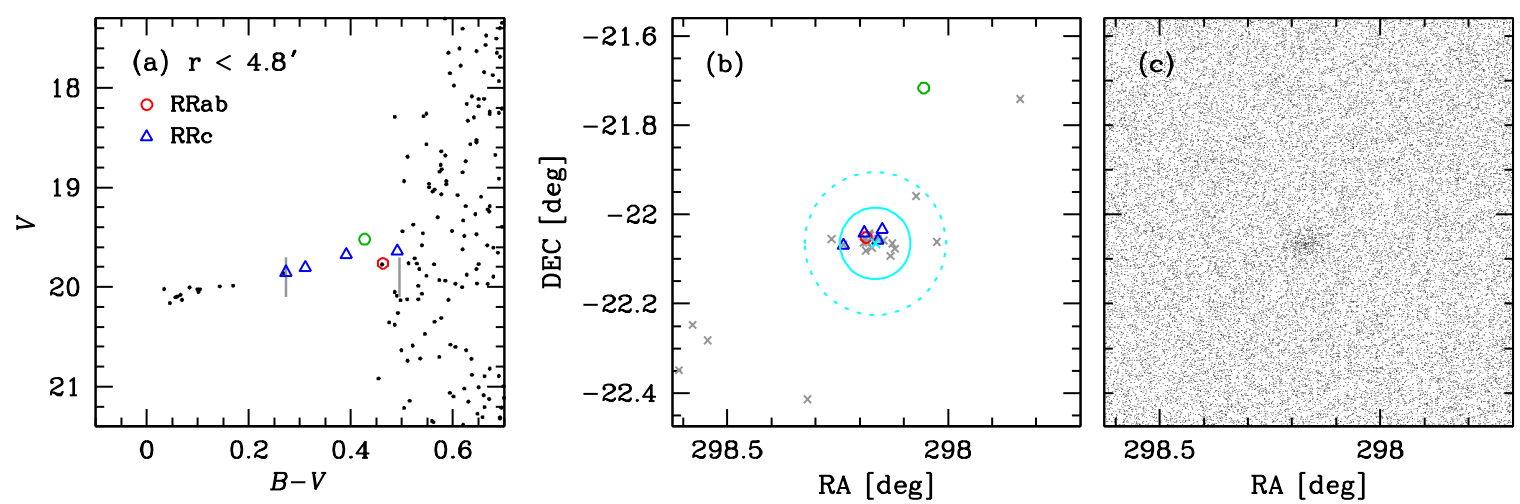

Figure 3. (a) CMD of stars within 4'.8 $\left(3 r_{h}\right)$ zoomed around the HB region, (b) spatial distribution of the RR Lyrae (small circles and triangles) and blue HB (gray crosses) stars, and (c) same as (b) but for all point sources detected in the field. The blue HB stars are defined as stars in the ranges of $0.0<B-V<0.27$ and $19.5<V<20.3$. The two gray vertical lines in panel (a) represent the empirical instability strip from Walker (1998), which is reddened by 0.097 mag (see the text). The solid and dotted cyan circles in panel (b) indicate $3 r_{h}(4.8)$ and $6 r_{h}(9.6)$ regions, respectively. The RR Lyrae and blue HB stars are clearly clustered around the galaxy center (cyan cross) from Mutlu-Pakdil et al. (2018). It is natural to consider V4 (small green circle) as belonging to the MW halo. 


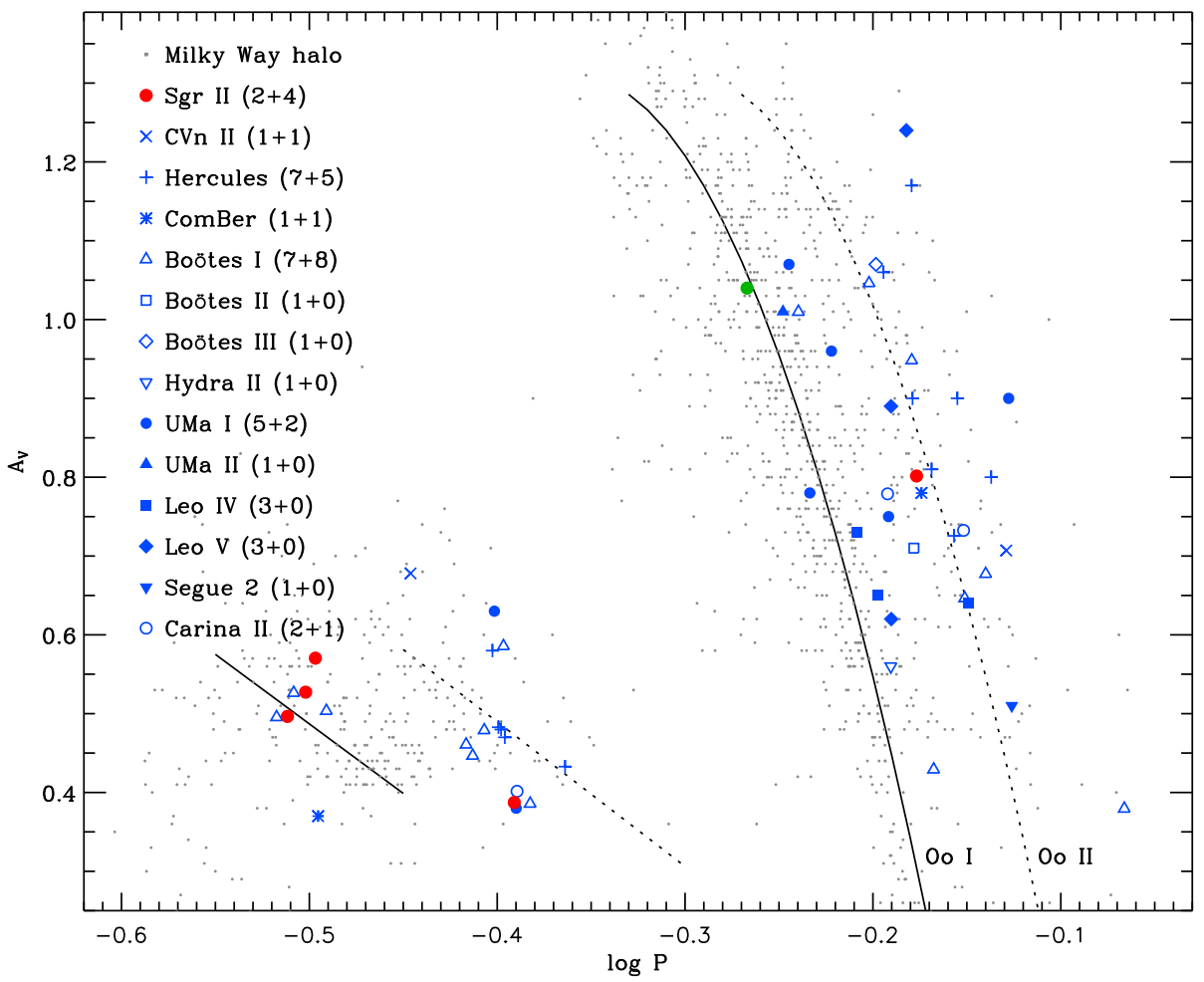

Figure 4. Period-amplitude diagram of the RR Lyrae stars in Sgr II (filled red circles), other 13 UFDs (blue symbols), and the MW halo (gray dots). The probable field star V4 is presented in the filled green circle. The solid and dotted lines are the mean distribution of RR Lyrae stars in the MW GCs that belong to Oo I and Oo II groups, respectively (Zorotovic et al. 2010; Cacciari et al. 2005). Numbers in parentheses indicate the numbers of $a b$ - and $c$-type RR Lyrae stars in each UFDs, separated by plus signs. This diagram is an update of Figure 10 in Vivas et al. (2016) and Figure 6 in Paper I. The RR Lyrae data were taken from Zinn et al. (2014) for the MW halo, Greco et al. (2008) for Canes Venatici II (CVn II), Musella et al. (2012) and Garling et al. (2018) for Hercules, Musella et al. (2009) for Coma Berenices (ComBer), Siegel (2006) for Boötes I, Vivas et al. (2016) for Hydra II, Garofalo et al. (2013) for Ursa Major I (UMa I), Moretti et al. (2009) for Leo IV, Medina et al. (2017) for Leo V, Boettcher et al. (2013) for Segue II, and Torrealba et al. (2018) for Carina II. In the case of Boötes II, Boötes III (Sesar et al. 2014), and UMa II (Dall'Ora et al. 2012), we used the data revised by Vivas et al. (2016), and the $A_{B}$ values in Boötes I were converted to $A_{V}$ using equations in Dorfi \& Feuchtinger (1999) following Vivas et al. (2016). The SDSS $g$-band amplitudes, $A_{g}$, of RR Lyrae variables in Hercules (Garling et al. 2018), Leo V, and Carina II, were transformed to $A_{V}$, adopting the equation, $V=g-0.59(g-r)-0.01$, in Jester et al. $(2005$, see their Table 1$)$, where for Leo V we assumed $(g-r)=0.15$ as the mean color of RRab stars without the SDSS $r$-band magnitude (see Paper I). 